\title{
Uso de bebederos artificiales por pequeños roedores en un bosque tropical seco en Oaxaca, México
}

\author{
Carlos A. Hernández-Gómez*iD, Gabriel P. Andrade-Ponce1(iD), Karen Lorena \\ Velásquez-Ciㄹ, Salvador MandujanoiD \\ 1 Posgrado, Instituto de Ecología A.C. Carretera Antigua a Coatepec 351, CP. 91073, Xalapa. Veracruz, México. \\ 2 Red de Biología y Conservación de Vertebrados, Instituto de Ecología A.C. Carretera Antigua a Coatepec 351, CP. 91073, Xa- \\ lapa. Veracruz, México. \\ * Correspondencia: carlos.hernandez@posgrado.ecologia.edu.mx
}

Resumen

La implementación de bebederos artificiales para fauna silvestre es una práctica de manejo común en regiones con escasez estacional de agua. En muchos casos los bebederos se instalan para beneficiar a especies de interés humano, sin embargo, éstos pueden ser usados por otras especies. En esta nota reportamos observaciones de pequeños roedores visitando los bebederos en una localidad en la Reserva de Biosfera Tehuacán-Cuicatlán. Se monitorearon los bebederos empleando 12 cámaras-trampa durante la temporada seca de 2018 en la localidad de San Gabriel Casa Blanca, Oaxaca. Se calculó la tasa de visita de roedores y se comparó entre dos tipos de bebederos. Se registraron 154 visitas de roedores de al menos cuatro géneros: Peromyscus, Liomys, Sigmodon y Dipodomys. No se encontraron diferencias en la tasa de visita promedio entre tipos de bebederos. Se sugiere implementar medidas como rampas o paredes cóncavas en los bebederos, que disminuyan el riesgo de ahogamiento para especies pequeñas. Son necesarios también otros estudios para evaluar el posible efecto de los bebederos sobre la biomasa, supervivencia y abundancia de roedores en bosques secos.

Palabras clave: enriquecimiento del hábitat, fototrampeo, pequeños mamíferos, recurso limitante, tasa de visita.

Abstract

The implementation of water developments for wildlife is a common management practice in regions with seasonal water shortages. In many cases, water developments are installed to benefit species of human interest, nevertheless, they can be used by other species. In this note we report observations of small rodents visiting water developments in a locality at the Tehuacán-Cuicatlán Biosphere Reserve. The water developments were monitored using 12 camera traps during the 2018 dry season in the locality of San Gabriel Casa Blanca, Oaxaca. We calculated the visit rate of rodents and compared it between two types of water developments. We registered 154 visits of rodents of at least four genera: Peromyscus, Liomys, Sigmodon and Dipodomys. We did not find significant differences between the visits among the water development types. We suggest to implement measures such as ramps or concave walls in the water developments, which reduce the risk of 
drowning for small species. Other studies are necessary to evaluate the possible effect of water developments on the biomass, survival and abundance of rodents in dry forests.

Key words: habitat enrichment, camera trap, visit rate, limiting resource, small mammals.

En zonas áridas y semiáridas el agua es un recurso limitante que influye sobre la distribución y abundancia de diferentes especies de vertebrados (Redfern et al. 2003; Muposhi et al. 2016), por lo que es común la implementación de estrategias de manejo como los bebederos artificiales (James et al. 1999; Smit \& Grant 2009; Ogutu et al. 2014). En México, la instalación de bebederos es una práctica común al interior de Áreas Naturales Protegidas y Unidades de manejo para la conservación de la vida silvestre (UMA), ya sea como medida de mitigación del estrés hídrico para la fauna en épocas de sequias (BorgesZapata et al. 2020) o para favorecer a especies cinegéticas como los ungulados (GastelumMendoza et al. 2014; Mandujano-Rodríguez \& Hernández 2019; Mandujano \& HernándezGómez 2020). Estos bebederos son aprovechados también por otros animales como carnivoros (Lynx rufus y Canis latrans), aves (Zenaida asiatica, entre otras), reptiles y anfibios (Destefano et al. 2000; O'Brien et al. 2006). No obstante, se cree que especies de menor tamaño, como los roedores, no hacen uso de bebederos debido al riesgo de ahogamiento o depredación (Bleich et al. 2006; Hall et al. 2013). En esta nota se describen observaciones incidentales del uso de bebederos artificiales por pequeños roedores en un bosque tropical seco a través de la técnica de fototrampeo.

Los registros fotográficos se obtuvieron en la localidad de San Gabriel Casa Blanca (17³9'$18^{\circ} 53^{\prime} \mathrm{N}, 96^{\circ} 55^{\prime}-97^{\circ} 44^{\prime} \mathrm{W}$ ), ubicada en el municipio de San Antonio Nanahuatipam, en el estado de Oaxaca, dentro de la Reserva de la Biosfera Tehuacán Cuicatlán. Su extensión es de 5.900 ha, caracterizados por terrenos accidentados, en los que predomina el bosque tropical seco (Mandujano et al. 2016). El clima es caluroso, semidesértico, con una temperatura promedio anual de $24^{\circ} \mathrm{C}$ y lluvias en verano, con una precipitación anual de $438 \mathrm{~mm}$. La zona de estudio está constituida como una UMA extensiva para la conservación y aprovechamiento de venado cola blanca, Odocoileus virginianus (Mandujano et al. 2016), por lo que, como medida de enriquecimiento del hábitat, se instalaron 28 bebederos artificiales, distanciados en promedio $98 \mathrm{~m}$ y abarcando un área aproximada de $63 \mathrm{ha}$. Los bebederos son de dos tipos: el tipo I tiene forma cuadrada, mide $50 \times 40 \mathrm{~cm}$ y $50 \mathrm{~cm}$ de profundidad, está al nivel del suelo, y tiene una capacidad de 100 litros. El tipo II tiene forma circular, una profundidad de $40 \mathrm{~cm}$, está elevado $30 \mathrm{~cm}$ del suelo, y tiene una capacidad de 80 litros.

El muestreo se realizó de febrero a junio de 2018 y los registros se obtuvieron a través de la técnica de fototrampeo; con el objetivo de monitorear el uso de bebederos por mamíferos medianos y grandes. Se instalaron 12 cámaras trampa, seis en bebederos del Tipo I y seis en bebederos del Tipo II, y se consideraron como registros independientes las fotografias consecutivas con una diferencia de 10 minutos (Hofmann et al. 2016). La identificación de las especies se basó en características morfológicas externas registradas en muestreos de captura previos en la localidad (datos no publicados). Se calculó la tasa de visitas para cada tipo de bebedero como: tasa = (eventos/esfuerzo de muestreo) * 100. Para determinar las diferencias en la tasa de visitas por tipo de bebedero se realizó una prueba de t-Student. Los análisis se realizaron en el lenguaje R versión 3.4.3 (R Core Team 2017). 
Se obtuvieron 154 registros de roedores en los bebederos de las familias Heteromyidae (géneros Dipodomys y Liomys), y Cricetidae (géneros Peromyscus y Sigmodon) (Figura 1), con un esfuerzo de muestreo de 908 días/cámara y una tasa de visita promedio de 18,8 \pm 37,7 visitas/100 días. Las tasas de visita promedio fueron de 16,16 \pm 44,71 para bebederos de tipo I y 9,5 $\pm 14,91$ para los de tipo II (Figura 2). Sin embargo, no existieron diferencias significativas entre los tipos de bebederos $(t=0,66, g l=6,09, P>0,05)$.
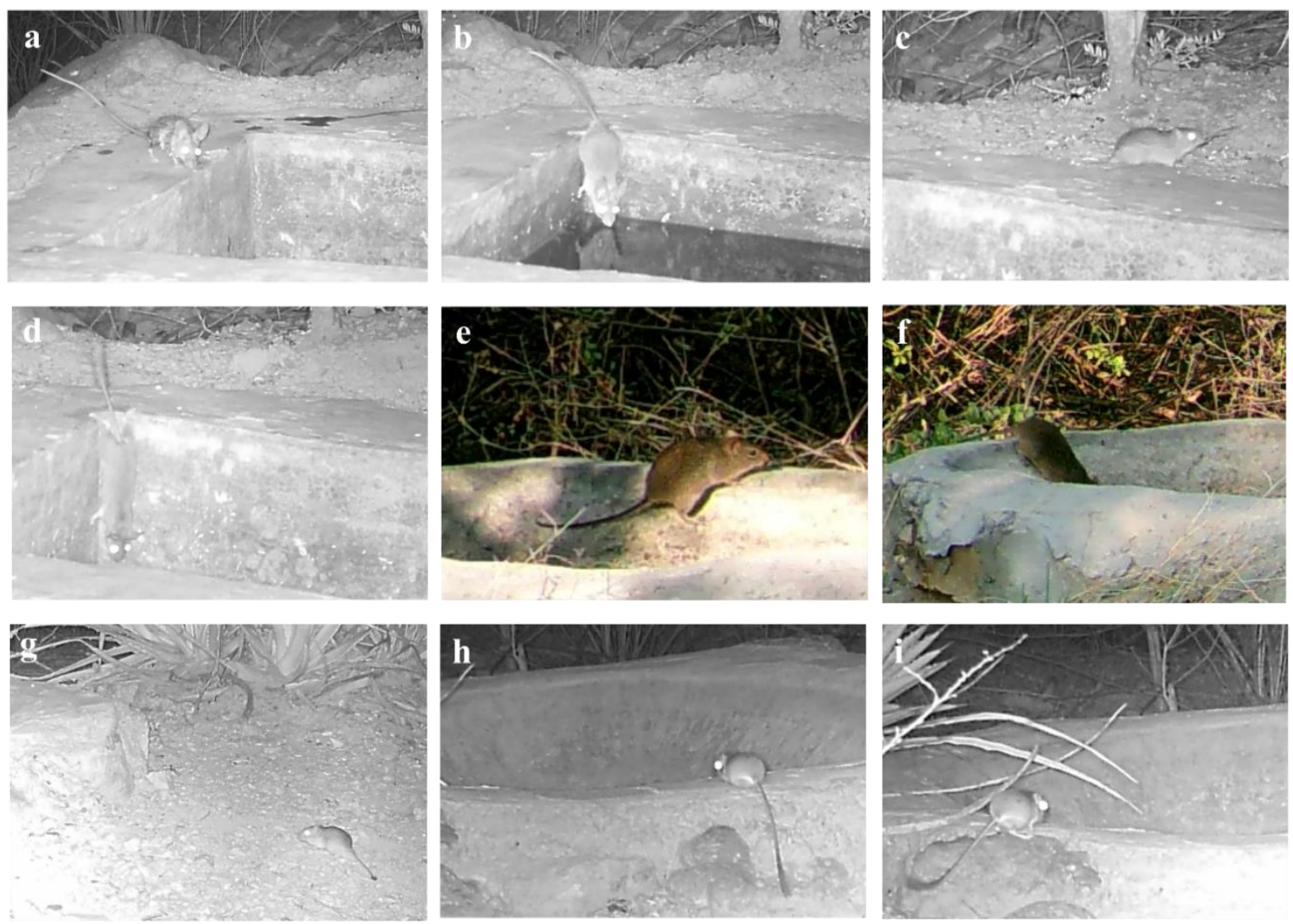

FIGURA 1. Registros fotográficos del uso de bebederos por roedores: $a, b$ y d) registro de un individuo del género Peromyscus.; c y g) individuo del género Liomys sp. en inmediaciones de un bebedero; e y f) individuo de la especie Sigmodon hispidus haciendo uso del bebedero; $h$, i) individuo del género Dipodomys en inmediaciones de bebederos.

La literatura establece que los roedores de zonas áridas no beben agua, debido a que la obtienen de su dieta (Brown 1973; Mares 1982; Morton 1985; Cortés et al. 2000). No obstante, en esta nota evidenciamos que los roedores utilizan los bebederos para beber o forrajear en las cercanías. De los roedores identificados, solo Peromyscus sp., y Sigmodon hispidus hicieron uso del recurso hídrico, mientras que Liomys sp. y Dipodomys sp. solo visitaron las cercanías de los bebederos, posiblemente porque estas últimas presentan adaptaciones fisiológicas que les permiten subsistir bajo condiciones de estrés hídrico (MacMillen 1983; MacMillen \& Hinds 1983).

De las visitas registradas fue posible evidenciar ejemplares de Peromyscus sp. colgándose para utilizar estos bebederos, e incluso, un ejemplar cayendo dentro (Figura 1a). Lo anterior puede suponer un riesgo debido a que la forma de los bebederos de Tipo I impide 
la salida de roedores a niveles bajos de agua, lo cual resulta en la muerte de los mismos. Pese al riesgo, no existieron diferencias en la tasa de visita por tipo de bebedero (Figura 2). Por lo anterior, una de las medidas propuestas para reducir el riesgo de los roedores al usar estos bebederos es instalar rampas que faciliten la salida de los organismos (Bleich et al. 2006), como la forma cóncava de los bebederos de tipo II (Figura 1f).

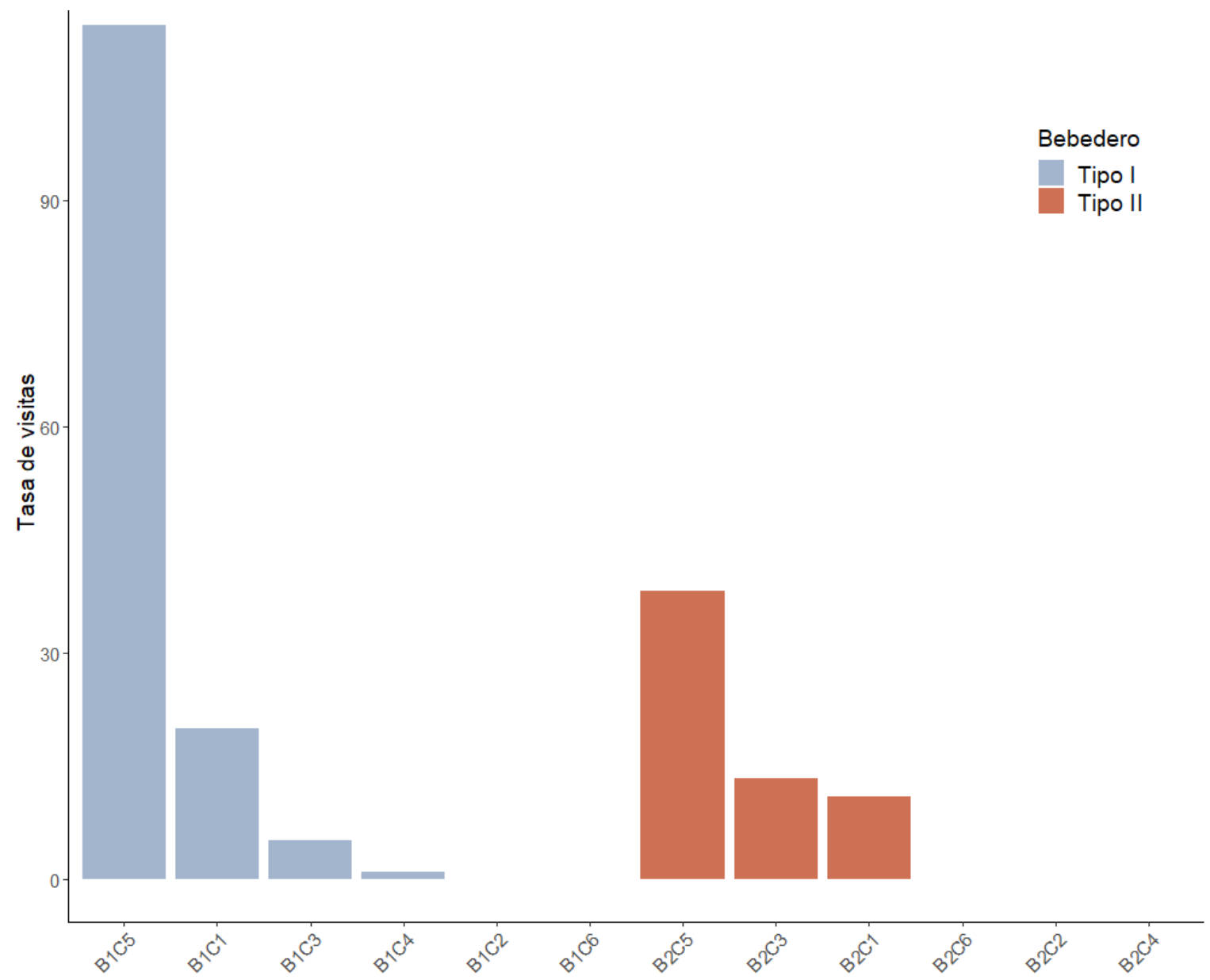

FIGURA 2. Tasa de visita de pequeños roedores en los bebederos artificiales. Los primeros seis bebederos (B1) corresponden a los registros en bebederos de tipo I, los últimos seis (B2) corresponden a los registros en bebederos de tipo II.

Dado que las cámaras se instalaron con el objetivo de monitorear especies de mayor tamaño, solo en algunos bebederos fue posible evidenciar cuándo un roedor bebió o simplemente visitó la cercanía del bebedero. En ese sentido un estudio enfocado a pequeños mamíferos podría brindar más información sobre la frecuencia de uso de bebederos, y su posible efecto sobre la comunidad de roedores. Por otra parte, la tasa de visita de cada uno de los bebederos fue diferencial, registrándose la mayoría de las observaciones en el bebedero B1C5 (Figura 2). Es posible que la cercanía al bebedero y el ángulo en el que se instaló cada cámara influya sobre la detección de las especies (Glen et al. 2013). Sin embargo, no se descarta que características del sitio como la estructura o tipo de vegetación también influyan sobre la tasa de visita (Kluever et al. 2016). Futuros análisis son necesarios para explorar si las condiciones ambientales proporcionan más 
ante posibles

recursos para los ratones, cobertura de protección o de escape ante posibles depredadores.

Se recomienda que al momento de realizar medidas de enriquecimiento del hábitat se evalué su posible efecto sobre otras especies, como roedores y pequeños vertebrados (Bleich et al. 2006). Finalmente, pese a que se ha reportado que los bebederos no influyen sobre la abundancia de roedores de zonas desérticas, se desconoce si pueden generar un efecto sobre la estructura (equitatividad), biomasa y tasa de supervivencia de las comunidades roedores que habitan zonas áridas y semiáridas.

\section{AGRADECIMIENTOS}

El presente trabajo forma parte del proyecto CONACYT CB-2015-01-256549. El primer autor recibió apoyo del CONACYT a través de la beca No. 27763. El estudio recibió apoyo logístico de la Red de Biología y Conservación de Vertebrados del Instituto de Ecología A.C. Agradecemos el apoyo en campo de A. Zavaleta, los guías en campo R. Mendoza y S. Chávez, así como el apoyo de la comunidad de San Gabriel Casa Blanca, y la asistencia de R. Rodríguez.

\section{REFERENCIAS}

Bleich V, Nelson S, Wood P, Wood H, Noles R. 2006. Retrofitting Gallinaceous Guzzlers to Enhance Water Availability and Safety for Wildlife. Wildlife Society Bulletin. 34(3):633-636. http://dx.doi.org/10.2193/0091-7648(2006)34

Borges-Zapata JY, Contreras-Moreno FM, Serrano-MacGregor I, Sima-Pantí DE, Coutiño-Cal y Mayor C, Zúñiga-Morales JA, Duque-Moreno VD, Hernández-Pérez EL, López-Chan JA. 2020. Uso de bebederos artificiales por el sereque centroamericano (Dasyprocta punctata) en la reserva de la biosfera de Calakmul, México. Agroproductividad. 13(1):51-58. http:// dx.doi.org/10.32854/agrop.vi0.1575

Brown JH. 1973. Species diversity of seed-eating desert rodents in sand dune habitats. Ecology 54(4):775-787. https:// doi.org/10.2307/1935672

Cortés A, Rosenmann M, Bozinovic F. 2000. Water economy in rodents: evaporative water loss and metabolic water production. Revista Chilena de Historia Natural. 73:311-321. http://dx.doi.org/10.4067/S0716-078X2000000200006

Destefano S, Schmidt SL, James C, deVos J. 2000. Observations of predator activity at wildlife water developments in Southern Arizona. Journal of Range Management. 53(3):255-258. http:// dx.doi.org/10.2307/4003428

Gastelum-Mendoza FI, Arroyo-Ortega JP, León-López LI. 2014. Estimación de la abundancia poblcional de fauna silvestre, mediante el uso de cámaras trampa. Agroproductividad 7(5):32-36.

Glen AS, Cockburn S, Nichols M, Ekanayake J, Warburton B. 2013. Optimising camera traps for monitoring small mammals. PLoS One 8(6). http:// dx.doi.org/10.1371/journal.pone.0067940

Hall LK, Larsen RT, Knight RN, Bunnell KD, McMillan BR. 2013. Water developments and canids in two North American deserts: A test of the indirect effect of water hypothesis. PLoS One 8(7). http:// dx.doi.org/10.1371/journal.pone.0067800

Hofmann GS, Coelho IP, Bastazini VAG, Cordeiro JLP, de Oliveira LFB. 2016. Implications of climatic seasonality on activity patterns and resource use by sympatric peccaries in northern Pantanal. International Journal of Biometeorology. 60(3):421-433. http://dx.doi.org/10.1007/s00484-015-1040-8 
James CD, Landsberg J, Morton SR. 1999. Provision of watering points in the Australian arid zone : a review of effects on biota. Journal of Arid Environments. 41(1):87-121. https:// doi.org/10.1006/jare.1998.0467

Kluever BM, Gese EM, Dempsey SJ. 2016. The influence of wildlife water developments and vegetation on rodent abundance in the Great Basin Desert. Journal of Mammalogy 97(4):1209-1218. http://dx.doi.org/10.1093/jmammal/gyw077

MacMillen RE. 1983. Adaptive physiology of heteromyid rodents. Great Basin Naturalist Memoirs. 7:65-76.

MacMillen RE, Hinds DS. 1983. Water regulatory efficiency in heteromyid rodents: A model and its application. Ecology 64(1):152-164. https:// doi.org/10.2307/1937337

Mandujano-Rodríguez S, Hernández C. 2019. Use of water developments by white-tailed deer in an extensive AHU in the biosphere reserve Tehuacan-Cuicatlan, Mexico. Agroproductividad 12(6):37-42. http://dx.doi.org/10.32854/agrop.v0i0.1406

Mandujano S, Hernández-Gómez CA. 2020. Use of artificial drinking containers by collared peccary during the dry season in a semi-arid tropical habitat in Central Mexico. Suiform Sound 8:1119. http:/ / dx.doi.org/10.4324/9781315111988-14

Mandujano S, López-Téllez MC, Barrera-Salazar A, Romero-Castañón S, Ramírez-Vera B, López-Tello E, Yañez-Arenas CA, Castillo-Correo JC. 2016. UMA extensiva de venado cola blanca en San Gabriel Casa Blanca, en la región de la Cañada. In: Mandujano S, editor. Venado cola blanca en Oaxaca. Potencial, conservación, manejo y monitoreo. Xalapa, México: Instituto de Ecología, A. C., Comisión Nacional para el Conocimiento de la Biodiversidad. p. 143-158.

Mares MA. 1982. Desert rodent adaptation and community structure. Great Basin Naturalist Memoirs. 8:30-43.

Morton SR. 1985. Granivory in arid regions: Comparison of Australia with North and South America. Ecology 66(6):1859-1866. https:// doi.org/10.2307/2937381

Muposhi VK, Gandiwa E, Chemura A, Bartels P, Makuza SM, Madiri TH. 2016. Habitat heterogeneity variably influences habitat selection by wild herbivores in a semi-arid tropical savanna ecosystem. PLoS One 11(9). http:// dx.doi.org/10.1371/journal.pone.0163084

O'Brien C, Waddell RB, Rosenstock SS, Rabe MJ. 2006. Wildlife use of water catchments in Southwestern Arizona. Wildlife Society Bulletin. 34(3):582-591. https:/ / doi.org/10.2193/00917648(2006)34[582:WUOWCI]2.0.CO;2

Ogutu JO, Reid RS, Piepho HP, Hobbs NT, Rainy ME, Kruska RL, Worden JS, Nyabenge M. 2014. Large herbivore responses to surface water and land use in an East African savanna: Implications for conservation and human-wildlife conflicts. Biodiversity and Conservation. 23(3):573-596. http://dx.doi.org/10.1007/s10531-013-0617-y

R Core Team. 2017. R: A language and environment for statistical computing. https://www.rproject.org

Redfern JV, Grant R, Biggs H, Getz WM. 2003. Surface-water constraints on herbivore foraging in the Kruger National Park, South Africa. Ecology 84(8):2092-2107. https:/ / doi.org/10.1890/01-0625

Smit IPJ, Grant CC. 2009. Managing surface-water in a large semi-arid savanna park: Effects on grazer distribution patterns. Journal for Nature Conservation. 17(2):61-71. https://doi.org/10.1016/j.jnc.2009.01.001

Editor: Diego J. Lizcano

Recibido 2020-07-27

Revisado 2020-08-10

Aceptado 2020-08-25

Publicado 2020-09-27 